\title{
Editorial
}

This issue illustrates the international relevance of the topics at the core of Health Expectations and demonstrates the array of different methods used to explore shared decision-making, patient-centred care and patient and public involvement.

The first three articles highlight the importance of qualitative methods. How people manage allergic rhinitis is explored through a qualitative and observational study as part of a randomized control trial in Australia. ${ }^{1}$ The researchers conclude that people on the whole did not and did not wish to rely on medication and that individualized support for selfmanagement can make a difference. The second article documents the development of a guidebook on osteoarthritis. ${ }^{2}$ Rather than framing the involvement process as research, Grime and Dudley present this as a collaboration based on sharing qualitative research papers creating a broader understanding than relying lived-experience alone. In the third article, Giacomini et $a l^{3}$ explore how university and community-based respondents in Ontario Canada interpret scenarios and options presented in a quantitative resource allocation survey that measured the effects of information types, goods and community sample on peoples' beliefs about fair resource allocation. They conclude that using a qualitative approach aids in understanding the processes, imagery and principled reasoning behind lay judgments of fair allocation.

Decision making in relation to quality links the next three articles. Fader et al. ${ }^{4}$ present a 'shopping' experiment on how people with incontinence would spend an individual budget. Participants were able to consider trade-offs between different levels of budget and different products. The conclusions suggest that providing an opportunity to control the purchase of their own supplies could improve patient satisfaction and result in a more efficient allocation of funds. Patient perceptions of the quality of primary care services in Greece were explored by Papanikolaou and Zygiaris. ${ }^{5}$ They apply an adapted version of SERVQUAL a service quality framework originally developed for a broad range of services such as banking and long-distance telephone services. The findings demonstrate the applicability of SERVQUAL to the measurement of quality in primary care services but also suggest that such generic measures may oversimplify patient's expectations. Broar, Nieboer and Bal present an ethnographic study of Dutch client participation in quality improvement programmes in mental health. ${ }^{6}$ Rather than identifying the exercise of power over clients by service providers, the findings suggest instances of mutual powerlessness as neither professionals nor clients knew how to ensure an involvement process beneficial for all. A more sophisticated approach to understanding power, they conclude is likely to lead to less defensiveness and better outcomes.

The importance of conceptual clarity is apparent in the next two articles. Hamrosi et al. ${ }^{7}$ used Australian focus-groups to explore needs and expectations relating to written medicines information. They found that this information was particularly important for 
people in rural areas with less access to physicians who are more reliant on pharmacists and written advice. Written information was an important source of reassurance but was not a substitute for spoken information from a healthcare professional. Researchers from McMaster University in Canada, McDonald et $a l^{8}$, present a framework for assessing the conceptual clarity and evidence base to support the development of quality standards in decision aids and particularly the International Patient Decision Aids Standards (IPDAS) Collaboration.

The next two articles also explore decisionmaking processes. Prostate cancer is the most common cancer in UK men excluding melanoma, and Sillence and Phoenix ${ }^{9}$ sought to understand whether those posting to four prostate cancer online forums were narrating systematic or non-systematic information processing in explaining their decision making. They conclude that there was more diversity in the approaches to decision making but that a third of the accounts stressed careful consideration of relevant information as central to the process. How British young people with degenerative conditions are involved in decisions about their own treatment and care is explored by Mitchell ${ }^{10}$ in semi-structured interview-based study. Ten disabled young people were each interviewed on three occasions and the majority reported feeling involved in the decision-making process that typically involved a complex process of 'tradeoffs' often involving the rejection of medical intervention to preserve independence from medical technology.

Jowsey et al. ${ }^{11}$ explored the sources of motivation for self-management using semi-structured interviews with 52 Australian adults with long-term conditions. They identified both external (health service professionals, family and friends) and internal (independence and wellness) sources of motivation as well as distinguishing between positive and negative motivations. They conclude that approaches to promoting self-management that focus primarily on the individual fail to take account the social context of motivations and demotivations that fluctuate and a key factor that limits self-management is financial hardship.

Citizen juries have been presented as a key mechanism to create deliberative involvement to inform prioritization but little is known of the short- or long-term impact of this resource intensive method. Drawing on questionnaires and interviews with members of two citizen juries with Canadian citizens Stafinsi et al. ${ }^{12}$ explore the impact of participation before, immediately following and 6 weeks after the deliberations. They provide clear evidence that participation shapes the views of participants and that the revised positions are retained for at least several weeks.

Health expectations publishes a wide range of articles from different national contexts and adopting varied methods but far fewer submissions engage with issues of policy. Prior with colleagues from Northern Ireland and the Republic of Ireland explore the relationship between policy and practice in the secondary prevention of coronary heart disease (CHD) in these two jurisdictions based on practitioner policy-maker interviews and the analysis of policy documents. ${ }^{13}$ Their interest was to understand better how policy might get into practice, and their findings illustrate that while there was agreement on the relevance of policies and operational documents to managing risk factors in CHD most primary care practitioners lacked awareness of the strategic documents. They conclude that politics is central to policy formation and implementation, policy documents signal and amplify awareness and that politicians need to mobilize resources and are a key determinant of health care.

As policies promoting choice and individual decision making continues to be at the heart of health reforms internationally, the final article is a reminder of the importance of linking policy and practice as well as practice to policy and that both are dependent on the presentation and framing of evidence. We need to ensure that we generate not just valid evidence but find ways of disseminating our findings in a way that makes an impact. 


\section{Jonathan Tritter}

Aston University

\section{References}

1 Smith L, Brown L, Saini B, Seeto C. Strategies for the management of intermittent allergic rhinitis: an Australian study. Health Expectations, 2014; 17: 153-162.

2 Grime J, Dudley B. Developing written information on osteoarthritis for patients: facilitating user involvement by exposure to qualitative research. Health Expectations, 2014; 17: 163-172.

3 Giacomini M, Hurley J, DeJean D. Fair reckoning: a qualitative investigation of responses to an economic health resource allocation survey. Health Expectations, 2014; 17: 173-184.

4 Fader MJ, Cottenden AM, Gage HM et al. Individual budgets for people with incontinence: results from a 'shopping' experiment within the British National Health Service. Health Expectations, 2014; 17: 185-195.

5 Papanikolaou V, Zygiaris S. Service quality perceptions in primary health care centres in Greece. Health Expectations, 2014; 17: 196-206.

6 Ickenroth AM, Grispen J, Ronda G et al. Motivation and experiences of self-testers regarding tests for cardiovascular risk factors. Health Expectations, 2014; 17: 207-218.
7 Hamrosi K, Aslani P, Raynor D. Beyond needs and expectations: identifying the barriers and facilitators to written medicine information provision and use in Australia. Health Expectations, 2014; 17: 219 230.

8 McDonald H, Charles C, Gafni A. Assessing the conceptual clarity and evidence base of quality criteria/standards developed for evaluating decision aids. Health Expectations, 2014; 17: 231-242.

9 Sillence E, Phoenix KH. Communicating health decisions: an analysis of messages posted to online prostate cancer forums. Health Expectations, 2014; 17: 243-252.

10 Mitchell W. Making choices about medical interventions: the experience of disabled young people with degenerative conditions. Health Expectations, 2014; 17: 253-265.

11 Jowsey T, Pearce-Brown C, Douglas K, Yen L. What motivates Australian health service users with chronic illness to engage in self-management behaviour? Health Expectations, 2014; 17: 266-276.

12 Stafinski T, Menon D, Yasui Y. Assessing the impact of deliberative processes on the views of participants: is it 'in one ear and out the other'? Health Expectations, 2014; 17: 277-289.

13 Prior L, Wilson J, Donnelly M et al. Translating policy into practice: a case study in the secondary prevention of coronary heart disease. Health Expectations, 2014; 17: 290-300. 